\title{
Una referencia global de almacenamiento de carbono en territorios colectivos
}

\author{
CONTRIBUCIONES DE LAS COMUNIDADES INDÍGENAS Y \\ LOCALES A LA MITIGACIÓN DEL CAMBIO CLIMÁTICO
}

Septiembre 2018

L

os bosques y otras tierras son esenciales para alcanzar las ambiciones climáticas y de desarrollo. Si se aprovecha adecuadamente, las soluciones climáticas naturales pueden contribuir con más del 37 por ciento de la mitigación rentable del $\mathrm{CO}_{2}$ para 2030,1 y la evidencia muestra que los Pueblos Indígenas y las comunidades locales son clave para lograr dichos resultados. Este informe presenta la evaluación más completa hasta la fecha del almacenamiento de carbono en tierras comunitarias documentadas en todo el mundo.

\section{Hallazgos principales}

Basándose en las estimaciones globales recientemente desarrolladas de la densidad de carbono para la biomasa de madera y los suelos asociados, el análisis de los datos de tenencia forestal más recientes de 64 países (que representan el 69\% de la cubierta forestal mundial) muestra que, en un mínimo:

1. Pueblos Indígenas y comunidades locales manejan al menos el 17 por ciento, 0293,061 millones de toneladas métricas (Mt) del carbono total almacenado en las tierras forestales de los países evaluados - una estimación global que es 5 veces mayor que la mostrada en un análisis previo de carbono forestal tropical, ${ }^{2}$ equivalente a 33 veces las emisiones de energía global de $2017 .{ }^{3}$

2. Veintidós por ciento $(217,991 \mathrm{MtC})$ del carbono forestal encontrado en los 52 países tropicales y subtropicales $^{4}$ en este análisis es administrado por las comunidades, y un tercio de este $(72,079 \mathrm{MtC}) \mathrm{se}$ encuentra en áreas donde los Pueblos Indígenas y las comunidades locales carecen de reconocimiento de sus derechos de tenencia - poniéndoles en riesgo a ellos, sus tierras y el carbono allí almacenado.

3. El carbono orgánico del suelo representa casi el 65 por ciento $(113,218 \mathrm{Mt})$ y casi el 90 por ciento (105,606 Mt) del carbono forestal total administrado por las comunidades en los países con bosques tropicales y no tropicales, ${ }^{5}$ respectivamente. Al proteger sus bosques y tierras, las comunidades no solo mantienen el carbono almacenado en los árboles (por encima y por debajo del suelo), sino también protegen vastos reservorios de carbono que, de otro modo, serían liberados a la atmósfera si los bosques superpuestos fueran destruidos.

4. El almacenamiento de carbono en territorios colectivos es mucho mayor y más extenso de lo que se puede evaluar a través de los datos disponibles. Esta evaluación sigue siendo una subestimación del carbono almacenado en los bosques colectivos en todo el mundo. Se desconoce la extensión total de los bosques y otras tierras en posesión de las comunidades indígenas y locales, y en particular aquellos en los que las comunidades aún no han logrado el reconocimiento legal de sus derechos, y aún no se cuenta con datos espacialmente explícitos sobre estas áreas. Por lo tanto, las vastas reservas de carbono dentro de tierras colectivas en países ricos en carbono como Indonesia y la República Democrática del Congo permanecen indocumentadas.
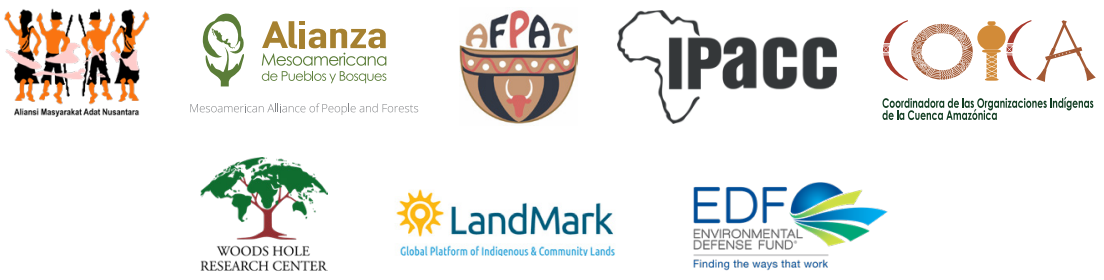

î. LandMark

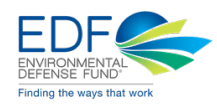




\section{Agradecimientos}

Este informe fue escrito, en orden alfabético, por Alain Frechette (La Iniciativa para los Derechos y Recursos), Chloe Ginsburg (La Iniciativa para los Derechos y Recursos) y Wayne Walker (El Centro de Investigación Woods Hole), con apoyo investigativo y analítico por Seth Gorelik (El Centro de Investigación Woods Hole), Stephanie Keene (La Iniciativa para los Derechos y Recursos), Chris Meyer (El Fondo de Defensa del Medio Ambiente), Katie Reytar (El Instituto de Recursos Mundiales) y Peter Veit (El Instituto de Recursos Mundiales).

Este producto fue desarrollado en estrecha colaboración con la Alianza de Pueblos Indígenas del Archipiélago (AMAN), la Alianza Mesoamericana de Pueblos y Bosques (AMPB), y la Coordinadora de las Organizaciones Indígenas de la Cuenca Amazónica (COICA). Está respaldado por la Asociación de Mujeres y Pueblos Indígenas de Chad (AFPAT) y el Comité Coordinador de los Pueblos Indígenas de África (IPACC). 


\section{Oportunidades y Desafíos}

La comunidad internacional necesita urgentemente reducir la deforestación y la degradación de los bosques, y mejorar el uso sostenible, la conservación y la restauración de los ecosistemas vitales. Sin embargo, la evidencia hasta la fecha muestra que los más cercanos y mejor posicionados para gestionar las tierras ricas en carbono de la Tierra, en especial los Pueblos Indígenas y las comunidades locales, aún no se han integrado a las soluciones climáticas nacionales y mundiales emergentes. Las tierras de los pueblos indígenas y de las comunidades locales no han sido plenamente reconocidas ni han recibido financiamiento adecuado, que reduce su capacidad para lograr su máximo potencial como una solución al cambio climático.

Hasta 2.500 millones de personas se ganan la vida en economías rurales a través de la administración de bosques comunitarios y otras tierras comunitarias que desempeñan un papel esencial en el mantenimiento de los servicios ecosistémicos a nivel de paisaje. En comparación con otros tipos de régimen, las comunidades con tenencia segura tienden a experimentar tasas más bajas de deforestación y emisiones de carbono forestal y a mantener niveles más altos de biodiversidad que producen paisajes más resilientes. ${ }^{6}$ Garantizar los derechos a la tierra de la comunidad es vital para la erradicación de la pobreza y la inseguridad alimentaria, el avance de la justicia de género y los esfuerzos para abordar la desigualdad y los conflictos relacionados con la tenencia.?

Lamentablemente, la capacidad de los Pueblos Indígenas y las comunidades locales para buscar medios de vida adaptados localmente y proteger ecosistemas de importancia mundial continúa siendo obstaculizada por el reconocimiento limitado de sus derechos de tenencia. A pesar de tener derechos sobre más de la mitad de la masa de tierra del mundo, los Pueblos Indígenas y las comunidades locales poseen legalmente solo el 10 por ciento de las tierras globales. ${ }^{8}$ De manera similar, de acuerdo con la última evaluación de 58 países que abarca casi el 92 por ciento de la masa forestal mundial, el progreso hacia el reconocimiento legal de los derechos de tenencia forestal comunitaria es totalmente inadecuado, representando poco más del 14 por ciento del área forestal a partir de $2017 .{ }^{\circ}$ Como resultado, las comunidades se enfrentan a amenazas crecientes de criminalización y violencia debido a la expansión continua de esquemas de uso de la tierra impulsados desde el exterior que no reconocen los derechos de tenencia de las comunidades o buscan de manera efectiva y abierta su consentimiento libre, previo e informado. ${ }^{10}$

Figura 1

\section{Al menos 293,061 millones de toneladas métricas de carbono (MtC)} se almacenan en las tierras forestales colectivas de los Pueblos Indígenas y las comunidades locales. « Esta cantidad es equivalente a 33 veces las emisiones de energía de 2017 a escala mundial »

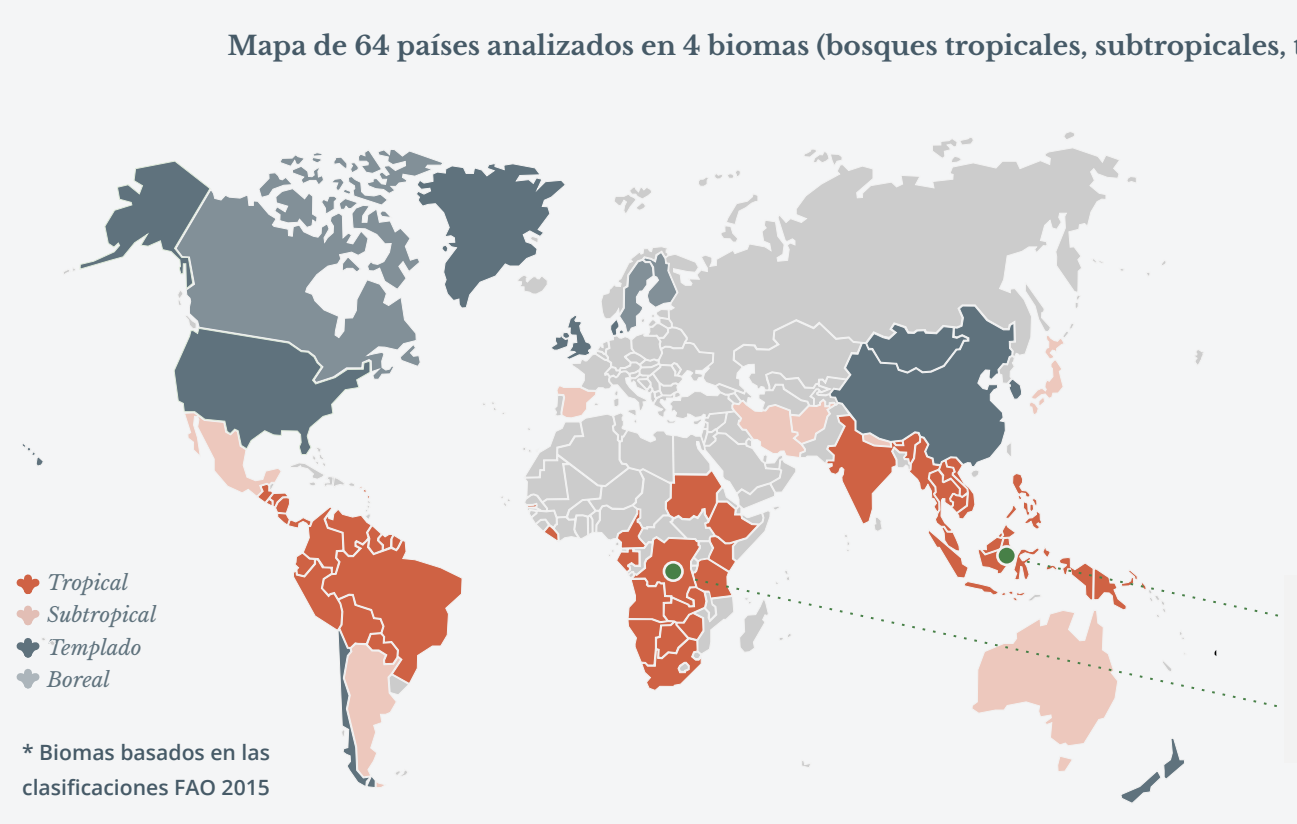
₹ Al menos un tercio del carbono manejado por la comunidad en los países tropicales y subtropicales analizados se encuentra en tierras forestales donde los pueblos indígenas y las comunidades locales carecen de reconocimiento legal de sus derechos de tenencia.

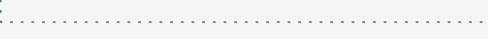
El almacenamiento de carbono en

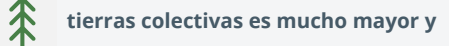
más extenso de lo que se puede evaluar a través de los datos disponibles. Se desconoce la extensión total de las tierras ocupadas por las comunidades indígenas y locales. Por lo tanto, las grandes reservas de carbono en países ricos en carbono como la República Democrática del Congo e Indonesia siguen sin documentarse.




\section{Almacenamiento de carbono en bosques colectivos de los Pueblos Indígenas y las comunidades locales, por país}

Nota: Los países se enumeran en orden descendente de la cantidad total de carbono almacenado en los bosques colectivos de los Pueblos Indígenas y las comunidades locales.

MtC = Millones de toneladas métricas de carbono

País

Brasil

China

Australia

República

Democrática del

Congo

México

Colombia

Papua Nueva

Guinea

Los Estados

Unidos

Canadá

Venezuela

Perú

Zambia

India

Groenlandia

Bolivia

Tanzania

Indonesia

Ecuador

Sudáfrica

Botsuana

Guatemala

Surinam

Zimbabue

Filipinas

Mongolia

Nicaragua

Panamá

Honduras

Namibia

Guyana
Carbono por encima y por debajo del suelo almacenado en tierras forestales colectivas bajo propiedad o designadas legalmente por comunidades (MtC)

17424

4016

1060

43

2837

5486

4596

1258

643

172

2947

3679

31

-

1495

1351

-

1169

481

90

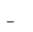

322

542

35

$-$

209

92

462
Carbono por encima y por debajo del suelo almacenado en tierras forestales colectivas que carecen de reconocimiento legal oficial (MtC)

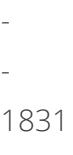

9076

117

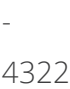

4322

982

1098

276

542

1444

21

\section{$-$}

65

447

1021

$-$

$$
-
$$

Carbono orgánico del suelo almacenado en tierras forestales colectivas legalmente reconocidas como bajo propiedad o designadas por comunidades (MtC)

21891

33238

8510

34

13643

9549

10439

11900

13551

241

6381

7208

178

$-$

2337

4196

-

2189

2557

1588

$-$

1733

1317

1615

$-$

495

$-$

1021

609
Carbono orgánico del suelo almacenado en tierras forestales colectivas que carecen de reconocimiento legal oficial (MtC)

13197

9907

1061

6898

1321

6393

5329

1174

3993

53

839

1871

1133

1120

501

1043 
Templado

Boreal

País

Camerún

Nepal

Irán

Suecia

Kenia

Nueva Zelanda

Costa Rica

Malasia

Vietnam

Guyana Francesa

chile

Irlanda

España

Belice

Paraguay

Inglaterra (Reino

Unido)

Liberia

Argentina

Japón

El Salvador

Tailandia

Camboya

Finlandia

Etiopia

Myanmar

Bután

Gabón

Sudan

Gambia

RPD Lao

Antigua y Barbuda

Corea del Sur

Afganistán

Angola

Total

51908 (MtC)

385

192

63

38

41

77

43

96

150

28

10

43

49

11

82

12

25

31

33

4

6

15

13

13

0.24

1

2

1

0.34

0.04
Carbono por encima

Carbono por encima y por debajo del suelo almacenado en tierras forestales colectivas bajo propiedad o designadas legalmente por comunidades

57

33

0.002 y por debajo del suelo almacenado en tierras forestales colectivas que carecen de reconocimiento legal oficial (MtC)
Carbono orgánico del suelo almacenado en tierras forestales colectivas legalmente reconocidas como bajo propiedad o designadas por comunidades (MtC)

580

594

631

585

539

502

146

172

255

282

154

266

244

196

105

155

123

86

76

51

40

37

18

29

7

4

3

1

162291
167

107

0.15
Carbono orgánico del suelo almacenado en tierras forestales colectivas que carecen de reconocimiento legal oficial (MtC) 


\section{Una Agenda Innovadora de Asociación e Investigación}

Este informe se basa en una asociación que expande, entre las organizaciones indígenas y comunitarias, científicos y expertos en políticas. Además de documentar la escala y la importancia de los sumideros y depósitos terrestres de gases de efecto invernadero administrados por comunidades en todo el mundo, la asociación busca llamar la atención mundial sobre la importancia de asegurar los derechos de tierras y recursos comunitarios, y hacer que los Pueblos Indígenas, las comunidades locales y las mujeres indígenas y rurales forman parte de las soluciones emergentes para el clima y el desarrollo.

Encabezado en $2014^{11}$ por una colaboración conjunta de los Coordinadores de las Organizaciones Indígenas de la Cuenca Amazónica (COICA), el Centro de Investigación Woods Hole (WHRC), la Red de Información Socioambiental Georeferenciada de la Amazonía (RAISG) y el Fondo de Defensa Ambiental (EDF), los análisis posteriores en $2015^{12}$ y $2016^{13}$ ampliaron la asociación para incluir a la Alianza Mesoamericana de Pueblos Indígenas y Bosques (AMPB), la Red de Pueblos Indígenas y Comunidades Locales para la Gestión Sostenible de los Ecosistemas Forestales en África Central (REPALAC), la Alianza de Pueblos Indígenas del Archipiélago (AMAN), la Iniciativa de Derechos y Recursos (RRI) y el World Resource Institute (WRI).

Utilizando datos de tenencia forestal de RRI, WRI y ONG participantes, investigaciones previas basadas en estimaciones de densidad de carbono sobre el suelo de alrededor del 2007 revelaron que bosques documentados indígenas y comunitarios en la región pantropical (37 países) representaban al menos 24 por ciento (54,546 Mt) del carbono total almacenado en la superficie en los bosques tropicales del mundo. ${ }^{14}$

\section{Rompiendo un nuevo terreno}

Por el contrario, este estudio combina puntos de datos más recientes, más zonas climáticas y más reservas de carbono, lo que demuestra que los Pueblos Indígenas y las comunidades locales administran 5 veces más carbono de lo que previamente se reconocía usando únicamente datos de carbono de bosques tropicales. Específicamente, presenta la primera evaluación de línea de base global de las reservas de carbono encontradas arriba y debajo del suelo, así como en la materia orgánica del suelo de tierras administradas por comunidades a través de zonas tropicales, subtropicales, templadas y boreales. Se basa en datos de densidad de carbono global recientemente generados de WHRC, datos de tenencia forestal actualizados de RRI, datos de tenencia de tierra espacialmente explícitos de LandMark y datos espaciales producidos recientemente a partir de una colaboración continua con AMAN para crear la evaluación más actual y precisa de carbono almacenado en tierras y bosques de comunidades indígenas y locales.

\section{Vincular Datos a Acciones}

La investigación realizada hasta la fecha sugiere que es poco probable que se logren esfuerzos internacionales para alcanzar los objetivos del Acuerdo de París y la Agenda 2030 para el Desarrollo Sostenible si no se garantizan los derechos de tenencia colectiva de los Pueblos Indígenas y las comunidades locales..$^{15}$ Como los principales administradores de algunos de los ecosistemas más biodiversos y ricos en carbono de la Tierra, los Pueblos Indígenas y las comunidades locales desempeñan un papel esencial en la gestión y protección de tierras y bosques ricos en carbono. En ausencia de esfuerzos concertados para asegurar legalmente sus derechos y fortalecer el acceso de la comunidad a los flujos de recursos técnicos y financieros, la capacidad de las comunidades indígenas y locales para mantener estos sistemas vitales está en riesgo. Como parte de los compromisos de los países de mantener el compromiso con la Plataforma de Comunidades Locales y Pueblos Indígenas en el contexto de las discusiones climáticas bajo la Convención Marco de las Naciones Unidas sobre el Cambio Climático (CMNUCC), los países también deben priorizar las acciones e inversiones nacionales que reconocen y aprovechan soluciones basadas en las comunidades de conservación de la tierra y los bosques en el contexto de las Contribuciones Determinadas a Nivel Nacional (CDN).

Basándose en el creciente cuerpo de evidencia que respalda este análisis, las alianzas indígenas y comunitarias que apoyan esta iniciativa hacen un llamado a los gobiernos individuales, la comunidad internacional, y los inversionistas y corporaciones a:

\section{Ampliar significativamente el reconocimiento de la tenencia basada en la comunidad, con un enfoque particular en reconocer a los Pueblos Indígenas y las comunidades locales como propietarios de sus tierras y bosques, aumentando el apoyo a países de bajos y medianos ingresos y}


organizaciones de la sociedad civil para implementar las leyes existentes y los procesos de reforma, y el avance de la legislación progresiva en el contexto de los compromisos climáticos y de desarrollo, como REDD + y los Objetivos del Desarrollo Sostenible (ODS).

2. Asegurar el consentimiento libre, previo e informado (CLPI) de los Pueblos Indígenas, las comunidades locales y las mujeres rurales e indígenas como parte de un ciclo continuo de compromiso entre las empresas, los inversores, los gobiernos y las instituciones de desarrollo.

3. Mejorar y ampliar continuamente el acceso de los Pueblos Indígenas y las comunidades locales al conocimiento crítico para fortalecer sus necesidades de defensa y apoyar el desarrollo sostenible y la resiliencia climática de las comunidades.

4. Dar prioridad a las inversiones bilaterales y multilaterales en iniciativas lideradas por indígenas y comunidades asociadas con los esfuerzos globales para reducir las emisiones derivadas de la deforestación y la degradación de los bosques y las tierras; fortalecer los esfuerzos de conservación y restauración basados en la comunidad; y mejorar las oportunidades de uso sostenible de la tierra / bosques.

5. Terminar con la criminalización y persecución de los Pueblos Indígenas y las comunidades locales que defienden sus tierras, bosques y recursos naturales.

\section{Métodos:}

El análisis estimó el almacenamiento de carbono en biomasa leñosa seca sobre el suelo, biomasa leñosa seca subterránea en vivo y materia orgánica del suelo en las tierras colectivas de los 64 países enumerados en la Figura 2 utilizando fuentes de datos espaciales y no espaciales disponibles. Los polígonos compatibles con el Sistema de Información Geográfica (SIG) (formato de geodatabase de archivos) que delinean los límites de las tierras colectivas se usaron en 40 de los 64 países. Los autores se basaron en datos de tenencia georeferenciados de LandMark: Ia Plataforma Global de Tierras Indígenas y Comunitarias para 36 de estos países con datos georreferenciados adicionales proporcionados por AMAN, la Unión Internacional para la Conservación de la Naturaleza (UICN) y Moabi. Los límites de la comunidad georeferenciados se combinaron con los datos de densidad de carbono ${ }^{16}$ a través del paquete de software estadístico R y se analizaron mediante un enfoque basado en vectores. En el caso de Indonesia, los límites de la comunidad georeferenciados disponibles (polígonos) se combinaron con los datos de densidad de carbono a través de un SIG (ArcGIS 10) y el personal técnico de AMAN Ios analizó mediante un enfoque basado en la trama.

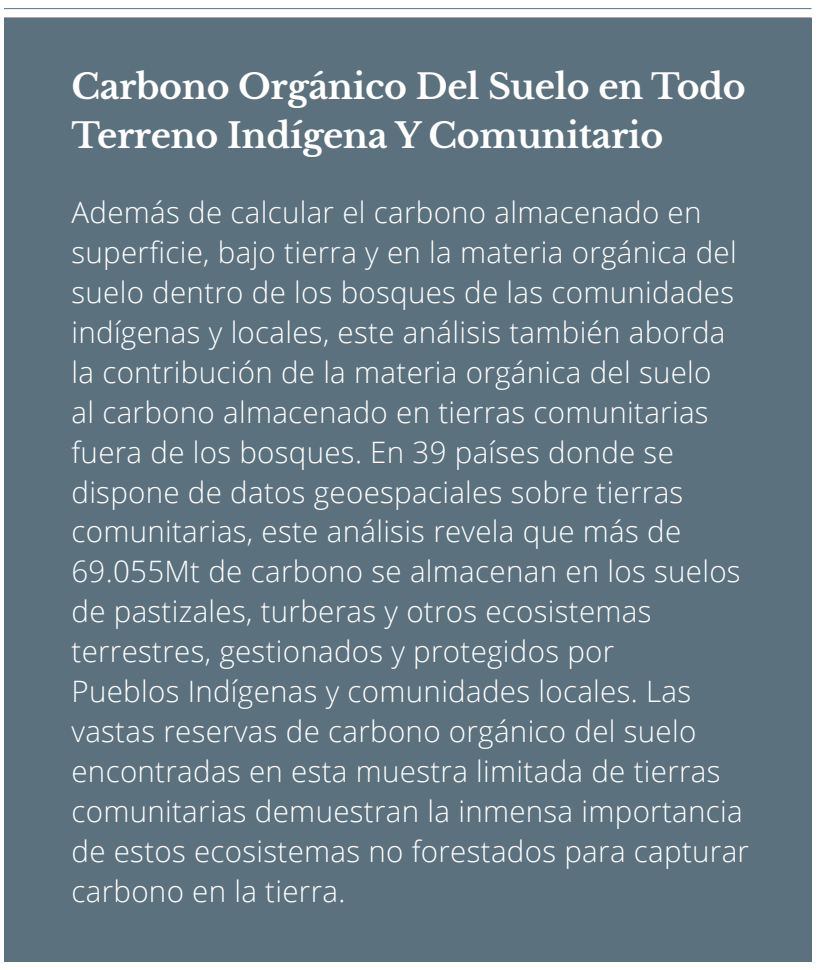
Terreno Indígena Y Comunitario

Además de calcular el carbono almacenado en superficie, bajo tierra y en la materia orgánica del suelo dentro de los bosques de las comunidades indígenas y locales, este análisis también aborda la contribución de la materia orgánica del suelo (bono almacenado en tierras comunitarias fuera de los bosques. En 39 países donde se dispone de datos geoespaciales sobre tierras comunitarias, este análisis revela que más de 69.055Mt de carbono se almacenan en los suelos pastizales, turberas y otros ecosistemas terrestres, gestionados y protegidos por Pueblos Indígenas y comunidades locales. Las vastas reservas de carbono orgánico del suelo encontradas en esta muestra limitada de tierras comunitarias demuestran la inmensa importancia de estos ecosistemas no forestados para captura carbono en la tierra.

Los países analizados incluyen espacialmente: Afganistán, Antigua y Barbuda, Australia, Belice, Bolivia, Botswana, Brasil, Canadá, Chile, Colombia, Costa Rica, República Democrática del Congo, Ecuador, El Salvador, Guayana Francesa, Groenlandia, Guatemala, Guyana, Honduras, Indonesia, Irán, Irlanda, Kenia, Malasia, México, Namibia, Nueva Zelanda, Nicaragua, Panamá, Paraguay, Perú, Filipinas, Sudáfrica, España, Suriname, Reino Unido, Estados Unidos de América, Venezuela, Zambia y Zimbabue. Los datos para estos países se consideran actuales e incluyen las tierras colectivas documentadas para áreas legalmente y no reconocidas legalmente. Sin embargo, aún existen brechas importantes en los datos y la cobertura. Por ejemplo, los resultados reportados en este análisis solo para Indonesia representan potencialmente menos de la mitad del carbono contenido en bosques comunitarios y otras tierras. Se realizarán esfuerzos en el futuro (anualmente o cuando los recursos lo permitan) para revisar las estimaciones contenidas en este documento a medida que la nueva información esté disponible en todo el mundo. 
Sin embargo, aún existen brechas importantes en los datos y la cobertura. Por ejemplo, los resultados reportados en este análisis solo para Indonesia representan potencialmente menos de la mitad del carbono contenido en bosques comunitarios y otras tierras. Se realizarán esfuerzos en el futuro (anualmente o cuando los recursos lo permitan) para revisar las estimaciones contenidas en este documento a medida que la nueva información esté disponible en todo el mundo.

Para los países que carecen de datos de tenencia espacialmente explícitos, el estudio incorporó datos legales de tenencia forestal basados en áreas de la base de datos de tenencia forestal mundial recientemente actualizada de RRI que incluye 58 países que cubren casi el 92 por ciento de los bosques del mundo a partir de 2017. ${ }^{17}$ Para estos países, cuantificado multiplicando las estimaciones de área conocidas para bosques colectivos por promedios nacionales de densidad de carbono. Se usaron capas político-administrativas para determinar los promedios nacionales de densidad de carbono. Este método se utilizó para Angola, Argentina, Bhután, Camboya, Camerún, China, Etiopía, Finlandia, Gabón, Gambia, India, Japón, Laos, Liberia, Mongolia, Myanmar, Nepal, Papúa Nueva Guinea, Corea del Sur, Sudán, Suecia, Tanzania, Tailandia y Vietnam. Se obtuvieron datos adicionales para bosques colectivos en India que se estima elegibles para el reconocimiento de derechos forestales comunitarios (pero aún no reconocidos) bajo la Ley de Derechos Forestales de 2006. Todos los resultados en este análisis representan una estimación mínima inicial del carbono almacenado en tierras forestales colectivas. Es probable que las contribuciones reales de los Pueblos Indígenas y las comunidades locales sean mucho más altas, y los resultados de esta evaluación se revisarán como nuevos datos espaciales y no espaciales concernientes a ambas áreas legalmente reconocidas como designadas y poseídas por los Pueblos Indígenas y las comunidades locales, como así como áreas colectivas que aún no han sido reconocidas legalmente, estén disponibles.

La WHRC estimó la densidad de carbono sobre el suelo utilizando mediciones de campo junto con información de los satélites de observación de la Tierra y del clima ancilar y de los datos del suelo. ${ }^{18}$ El resultado (no publicado) fue una estimación global continua basada en mapas de la cantidad y distribución de carbono almacenado en la biomasa seca y leñosa (hojas, ramas, tallos) de la vegetación de la Tierra para el período 2016 a una resolución espacial de 500 metros. ${ }^{19}$

La densidad de carbono subterránea se estimó mediante la aplicación de relaciones de raíces / brotes a nivel de bioma o ecorregión publicadas al mapa de densidad de carbono sobre el suelo. ${ }^{20}$ El resultado (sin publicar) fue una estimación basada en mapas de la cantidad y distribución de carbono almacenado bajo tierra en la biomasa seca leñosa (raíces) de la vegetación de la Tierra para el período de 2016 con una resolución espacial de aproximadamente 500 metros. La WHRC estimó la materia orgánica del suelo utilizando mediciones de campo junto con información sobre el uso de la tierra, características climáticas, topográficas, geológicas y de relieve. El resultado fue una estimación basada en mapas de la cantidad y distribución de carbono almacenado en la materia orgánica del suelo de la Tierra por encima de una profundidad de 2 metros para el marco temporal de 2010 a una resolución espacial de 10 kilómetros (remuestreado a 500 metros para consistencia). ${ }^{21}$ 


\section{Endnotes}

1 Griscom, Bronson W., and Justin Adams, Peter W. Ellis, Richard A. Houghton, Guy Lomax, Daniela A. Miteva, William H. Schlesinger, David Shoch, Juha V. Siikamäki, Pete Smith, Peter Woodbury, Chris Zganjar, Allen Blackman, João Campari, Richard T. Conant, Christopher Delgado, Patricia Elias, Trisha Gopalakrishna, Marisa R. Hamsik, Mario Herrero, Joseph Kiesecker, Emily Landis, Lars Laestadius, Sara M. Leavitt, Susan Minnemeyer, Stephen Polasky, Peter Potapov, Francis E. Putz, Jonathan Sanderman, Marcel Silvius, Eva Wollenberg, and Joseph Fargione. 2017. Natural climate solutions. PNAS (114) 44: 11645-1165. Available at: http://www.pnas.org/content/114/44/11645.

2 Rights and Resources Initiative, Woods Hole Research Center, and World Resources Institute. 2016. Towards a global baseline of carbon storage in collective lands. Rights and Resources Initiative: Washington, DC. Available at: https://rightsandresources.org/ en/publication/global-baseline-carbon-storage-collective-lands.

332.5 GtCO2 = 8.86 GtC. Data from: International Energy Agency. 2018. Global Energy \& CO2 Status Report 2017. International Energy Agency. Available at: https://www.iea.org/publications/freepublications/publication/GECO2017.pdf.

4 Countries were classified as tropical, subtropical, temperate, and boreal according to the ecological domain classifications used in the FAO Global Forest Resources Assessment 2015. FAO. 2016. FRA 2015 Process Document. Forest Resources Assessment Working Paper 186, Food and Agriculture Organization of the United Nations, Rome, 12-21. Available at: http://www.fao.org/3/abr632e.pdf.

5 Non-tropical forest countries include: subtropical, temperate, and boreal forest countries.

6 Ben Yishay, A., and S. Heuser, D. Runfola, and R. Trichler. 2017. Indigenous land rights and deforestation: evidence from the Brazilian Amazon. Journal of Environmental Economics and Management 86: 29-47; Blackman, A., and P. Veit. 2018. Titled Amazon Indigenous Communities Cut Forest Carbon Emissions. Ecological Economics 153: 56-67; Blackman, A., and L. Corral, E. Lima, and G. Asner. 2017. Titling indigenous communities protects forests in the Peruvian Amazon. PNAS 114: 123-128; RAISG, Ecociencia, Woods Hole Research Center, Instituto del Bien Común, EDF, Gaia Amazonas, and COICA. 2017. Amazonian Indigenous Peoples Territories and their Forests Related to Climate Change: Analyses and Policy Options. Available at: http://www.edf.org/sites/ default/files/indigenous-territories-barrier-to-deforestation.pdf; \& Stevens, Caleb, and Robert Winterbottom, Katie Reytar, and Jenny Springer. 2014. Securing Rights, Combating Climate Change: How strengthening community forest rights mitigates climate change. World Resources Institute and Rights and Resources Initiative, Washington, DC. Available at: http://www.wri.org/publication/securing-rights-combating-climate-change.

7 Rights and Resources Initiative. 2017. Securing Community Land Rights: Priorities \& Opportunities to Advance Climate \& Sustainable Development Goals. Rights and Resources Initiative, Washington, D.C. Available at: https://rightsandresources.org/en/ publication/securing-community-land-rights-rri-brief/\#sthash.MrbRjWoK.dpbs.

8 Rights and Resources Initiative. 2015. Who Owns the World's Land? A global baseline of formally recognized indigenous and community land rights. Rights and Resources Initiative, Washington, DC. Available at: https://rightsandresources.org/wp-content/ uploads/GlobalBaseline_web.pdf.

9 Rights and Resources Initiative. 2018. At a Crossroads: Trends in Recognition of Community-Based Forest Tenure from 20022017. Rights and Resources Initiative, Washington, DC. Available at https://rightsandresources.org/en/publication/at-a-crossroadstrends-in-recognition-of-community-based-forest-tenure-from-2002-2017/.

10 Tauli-Corpuz, Victoria. 2018. Report of the Special Rapporteur of the Human Rights Council on the rights of indigenous peoples. Report A/73/176, United Nations, New York. Available at: http://www.un.org/en/ga/search/view_doc.asp?symbol=A/73/176. 
11 Walker, Wayne, and A. Baccini, S. Schwartzman, S. Ríos, M. Oliveira-Miranda, C. Augusto, M. Romero Ruiz, C. Soria Arrasco, B. Ricardo, R. Smith, C. Meyer, J.C. Jintiach, and E. Vasquez Campos. 2014. Forest carbon in Amazonia: The unrecognized contribution of indigenous territories and protected natural areas. Carbon Management (5) 5-6: 479-485.

12 Woods Hole Research Center and Environmental Defense Fund. 2015. Tropical Forest Carbon in Indigenous Territories: A Global Analysis. Woods Hole Research Center, Woods Hole, Massachusetts. Available at: https://www.edf.org/sites/default/files/ tropical-forest-carbon-in-indigenous-territories-a-global-analysis.pdf.

13 Rights and Resources Initiative, Woods Hole Research Center, and World Resources Institute 2016.

14 Rights and Resources Initiative 2016.

15 Rights and Resources Initiative 2017.

16 Data adapted for aboveground and belowground carbon from: Baccini, Alessandro and W. Walker, L. Carvalho, M. Farina, D. Sulla-Menashe, and R.A. Houghton. 2012 and 2017. Tropical forests are a net carbon source based on aboveground measurements of gain and loss. Science 358(6360), 230-234.; Data adapted for soil organic matter from Sanderman, Jonathan, Tomislav Hengl, and Gregory J. Fiske. Soil carbon debt of 12,000 years of human land use. PNAS 114 (36): 9575-9580.

17 Rights and Resources Initiative 2018.

18 The data set was generated using field measurements co-located with (a) satellite-based Light Detection and Ranging (LiDAR) observations from the NASA Geoscience Laser Altimeter System (GLAS) for the tropics and (b) airborne-Lidar based biomasS allometric equations outside of the tropics together with a 2016 cloud-free temporal mosaic generated from NASA Moderate Resolution Imaging Spectrometer (MODIS) and Nadir Adjusted Reflectance (NBAR) data.

19 For further information on the methods used to produce the aboveground biomass data set: Baccini, Alessandro, and S.J. Goetz, W.S. Walker, N.T. Laporte, M. Sun, D. Sulla-Menashe, J. Hackler, P.S. A. Beck, R. Dubayah. M.A. Friedl, S. Samanta, and R.A. Houghton.. 2012. Estimated carbon dioxide emissions from tropical deforestation improved by carbon-density maps. Nature Climate Change 2(3):.182-185; and Baccini et al. 2017.

20 Root:shoot ratios taken from: Hutchison, James, and Andrea Manica, Ruth Swetnam, Andrew Balmford, and Mark Spalding. 2014. Predicting Global Patterns in Mangrove Forest Biomass. Conservation Letters 7(3): 233-240; and Mokany, Carol and R. John Raison, and Anatoly S. Prokushkin. 2006. Critical analysis of root:shoot ratios in terrestrial biomes. Global Change Biology. Regional ecosystem (biome) boundaries based on: Dinerstein, Eric, and, David Olson, Anup Joshi, Carly Vynne, Neil D. Burgess, Eric Wikramanayake, Nathan Hahn, Suzanne Palminteri, Prashant Hedao, Reed Noss, Matt Hansen, Harvey Locke, Erle C Ellis, Benjamin Jones, Charles Victor Barber, Randy Hayes, Cyril Kormos, Vance Martin, Eileen Crist, Wes Sechrest, Lori Price, Jonathan E. M. Baillie, Don Weeden, Kierán Suckling, Crystal Davis, Nigel Sizer, Rebecca Moore, David Thau, Tanya Birch, Peter Potapov, Svetlana Turubanova, Alexandra Tyukavina, Nadia de Souza, Lilian Pintea, José C. Brito, Othman A. Llewellyn, Anthony G. Miller, Annette Patzelt, Shahina A. Ghazanfar, Jonathan Timberlake, Heinz Klöser, Yara Shennan-Farpón, Roeland Kindt, Jens-Peter Barnekow Lillesø, Paulo van Breugel, Lars Graudal, Maianna Voge, Khalaf F. Al-Shammari, and Muhammad Saleem. 2017. An Ecoregion-Based approach to protecting half the terrestrial realm. BioScience 67(6), 534-545. Where ecoregional root:shoot assignments could not be readily made, belowground carbon density was estimated using Equation 1 from Mokaney et al. 2006.

21 For further information on the methods used to produce the soil organic matter data set: Sanderman et al. 2017. 


\section{La Iniciativa para los Derechos y Recursos}

RRI es una coalición mundial de 15 socios principales, 7 redes afiliadas, 14 miembros internacionales y más de 150 organizaciones colaboradoras a nivel internacional, regional y comunitario, que se dedica a fomentar los derechos de los Pueblos Indígenas y las comunidades locales sobre la tierra y los recursos forestales. La RRI aprovecha las capacidades y los conocimientos de los miembros de la coalición para promover la seguridad de los derechos locales sobre la tierra y los recursos y para impulsar reformas políticas y de mercado progresistas.

La RRI opera bajo la coordinación del Grupo para los Derechos y Recursos, organización sin ánimo de lucro con sede en Washington, D.C. Si desea obtener más información, visite: www.rightsandresources.org/es.

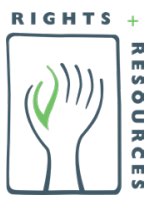

2715 M St NW

Suite 300

Washington, DC 20007

www.rightsandresources.org/es @RightsResources

\section{Socios}
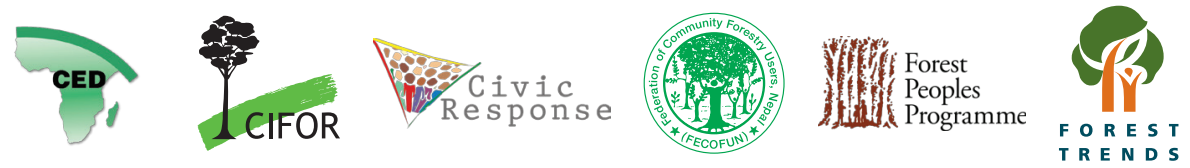

\section{HELVETAS}
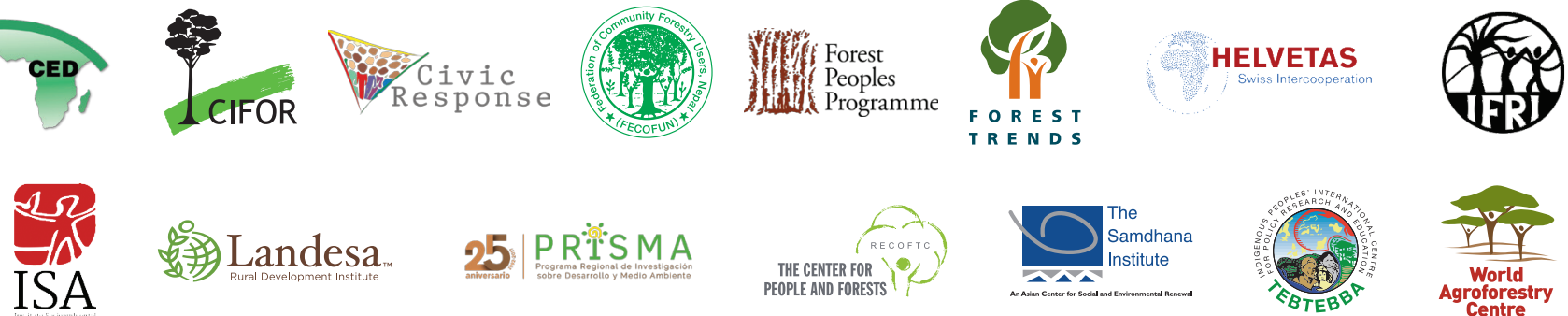

25.
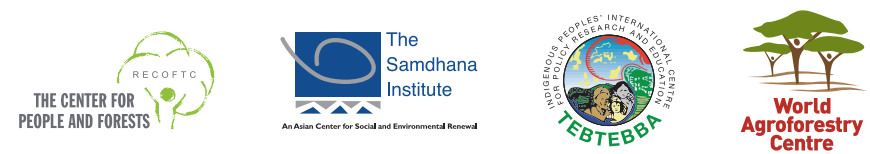

\section{Redes afiliadas}
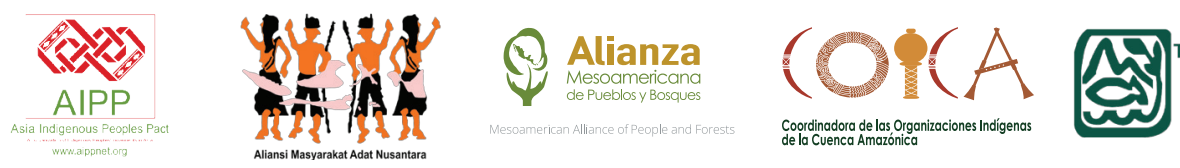

The International
Association
for the Study of
the Commons
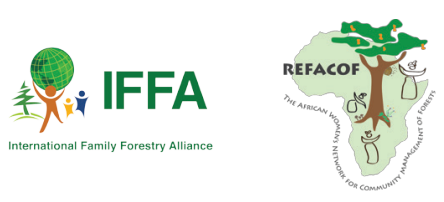

\section{Patrocinadores}

